\title{
Prediction of 90-Day Mortality among Sepsis Patients Based on a Nomogram Integrating Diverse Clinical Indices
}

\author{
Qingbo Zeng $\mathbb{D}^{1,2}$ Longping He, ${ }^{1}$ Nianqing Zhang, ${ }^{2}$ Qingwei Lin, ${ }^{1}$ Lincui Zhong, \\ and Jingchun Song $\mathbb{D}^{1}$ \\ ${ }^{1}$ Intensive Care Unit, The 908th Hospital of Chinese PLA Logistical Support Force, Nanchang, China \\ ${ }^{2}$ Intensive Care Unit, Nanchang Hongdu Hospital of Traditional Chinese Medicine, Nanchang, China \\ Correspondence should be addressed to Jingchun Song; songjingchun@126.com
}

Received 4 June 2021; Accepted 8 October 2021; Published 20 October 2021

Academic Editor: Washington L. C. dos Santos

Copyright ( 2021 Qingbo Zeng et al. This is an open access article distributed under the Creative Commons Attribution License, which permits unrestricted use, distribution, and reproduction in any medium, provided the original work is properly cited.

\begin{abstract}
Background. Sepsis is prevalent among intensive care units and is a frequent cause of death. Several studies have identified individual risk factors or potential predictors of sepsis-associated mortality, without defining an integrated predictive model. The present work was aimed at defining a nomogram for reliably predicting mortality. Methods. We carried out a retrospective, single-center study based on 231 patients with sepsis who were admitted to our intensive care unit between May 2018 and October 2020. Patients were randomly split into training and validation cohorts. In the training cohort, multivariate logistic regression and a stepwise algorithm were performed to identify risk factors, which were then integrated into a predictive nomogram. Nomogram performance was assessed against the training and validation cohorts based on the area under receiver operating characteristic curves (AUC), calibration plots, and decision curve analysis. Results. Among the 161 patients in the training cohort and 70 patients in the validation cohort, 90-day mortality was $31.6 \%$. Older age and higher values for the international normalized ratio, lactate level, and thrombomodulin level were associated with greater risk of 90-day mortality. The nomogram showed an AUC of 0.810 (95\% CI 0.739 to 0.881 ) in the training cohort and 0.813 ( $95 \%$ CI 0.708 to 0.917 ) in the validation cohort. The nomogram also performed well based on the calibration curve and decision curve analysis. Conclusion. This nomogram may help identify sepsis patients at elevated risk of 90-day mortality, which may help clinicians allocate resources appropriately to improve patient outcomes.
\end{abstract}

\section{Introduction}

Sepsis is life-threatening organ dysfunction initiated by the body's overwhelming response to infection [1]. Although significant advances have been made in intensive care and supportive technology to treat sepsis, it remains associated with high morbidity and mortality. The global incidence rate is around 437 per 100000 person-years, and approximately $17 \%$ of sepsis cases die in hospital [2]. These figures are even higher in China, where up to $20 \%$ of patients in intensive care units have sepsis [3].

The pathogenesis of sepsis is complex and involves coagulation disorder, inflammation imbalance, immune dysfunction, and mitochondrial and endothelial damage [4]. Better understanding of the disease's pathophysiology and identifi- cation of reliable predictors of short-term mortality are critical for guiding interventions and improving prognosis.

Several studies have analyzed risk factors for mortality in patients with sepsis [5-9], but most have focused on biomarkers related to inflammation or the function of certain organs. For such a complex disease, prediction algorithms may need to take a range of biomarkers into account. Therefore, the main objective of the present study was to consider a diversity of potential clinicodemographic factors for constructing a nomogram to predict 90-day mortality in sepsis.

\section{Materials and Methods}

2.1. Patient Selection and Data Collection. This retrospective study examined electronic medical record from a consecutive 


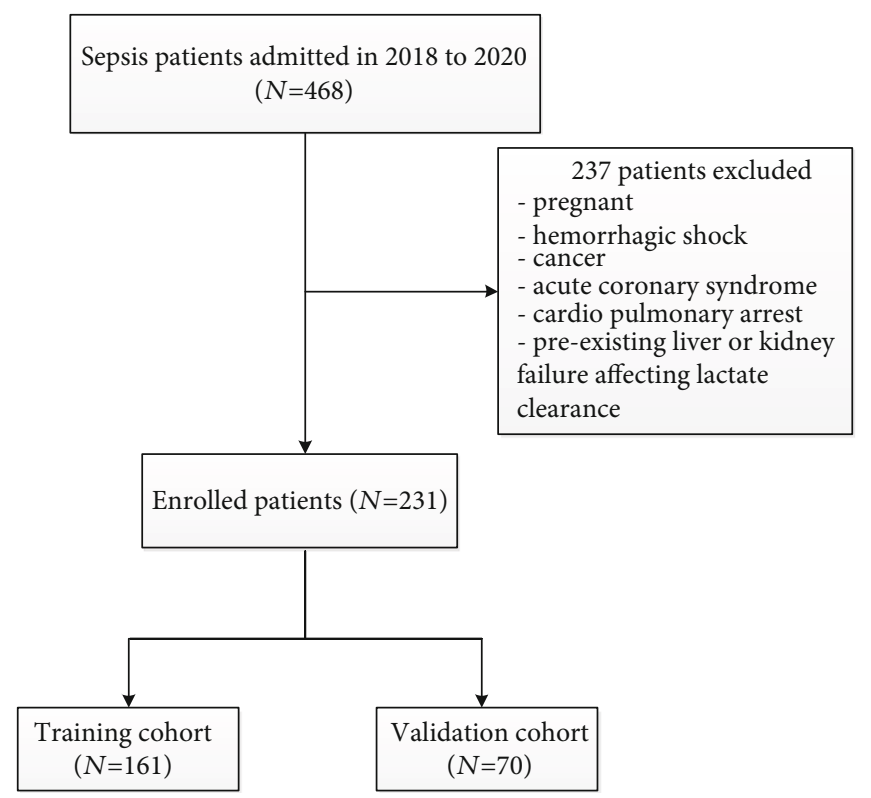

FIGURE 1: Flowchart of patients excluded by each criterion.

sample of 231 patients who had been diagnosed with sepsis and had been admitted to the intensive care unit at the 908 ${ }^{\text {th }}$ People's Liberation Army Hospital (Nanchang, China) between May 2018 and October 2020. A flowchart of patients excluded by each criterion is shown in Figure 1. To be enrolled in the study, patients had to be older than 17 years and diagnosed with sepsis according to the Third International Consensus Definition for Sepsis ("Sepsis-3") [10]: infection had to be confirmed through culture tests and the Sequential Organ Failure Assessment (SOFA) score had to be at least 2 [4]. Patients were excluded if they were pregnant or had a history of hemorrhagic shock, cancer, acute coronary syndrome, or cardiopulmonary arrest. This study was approved by the Ethics Committee of the $908^{\text {th }}$ People's Liberation Army Hospital with a waiver of informed consent. Baseline demographic data (age, sex) were collected, as were data on the site of infection, comorbidities, 90-day mortality, and severity of illness, based on the Acute Physiology and Chronic Health Evaluation II (APACHE II) score [11] and the SOFA scores [12] on the first day of admission to the intensive care unit, as well as numerous laboratory and clinical variables which were obtained four hours after admission (see Table 1).

2.2. Statistical Analysis and Nomogram Construction. All statistical analyses were performed using R 4.0.1 (R Core Team, Vienna, Austria) and SPSS 25.0 (IBM, Chicago, IL, USA). Differences associated with a two-sided $P<0.05$ were considered statistically significant. Data for continuous variables were presented as mean \pm standard deviation or as median (interquartile range (IQR)). Differences between groups were assessed for significance using Student's $t$-test in the case of normally distributed data or using the Mann-Whitney test in the case of a skewed distribution. Data for categorical variables were expressed as counts and percentages, and differences were assessed using $\chi^{2}$ or Fisher's exact test. The variance inflation factor (VIF) was used to test collinearity between continuous variables, and an arithmetic square root of VIF $\leq 10$ was regarded as noncollinearity. Patients were randomized into training and validation cohorts in a ratio of $2: 1$. Clinical variables in the training cohort were entered into multivariate logistic regression, and backward stepwise selection was applied using the likelihood ratio test and Akaike's information criterion as the stopping rule [13]. The regression results from the training cohort were used to define a nomogram to predict 90-day mortality. The same regression equations for the training cohort were also applied to the data for the validation cohort in order to verify the nomogram. Calibration curves, accompanied by the Hosmer-Lemeshow test, were used to evaluate the predictive model. Its discriminative ability was assessed based on the area under the receiver operating characteristic curve (AUC). For clinical usefulness, net benefit was examined against the training and validation cohorts using decision curve analysis (DCA).

\section{Results}

3.1. Baseline Characteristics of Patients with Sepsis. Among the 231 patients in the study, $61.9 \%$ were men, the median age was 70 years (range, 18 to 96 years), and 73 (31.6\%) died within 90 days of follow-up. In both the training and validation cohorts, patients who survived for 90 days had significantly lower levels of many clinical variables than those who died (Table 1), including tissue plasminogen activatorinhibitor complex, thrombin-antithrombin complex, prothrombin time, international normalized ratio, activated partial thrombin time, thrombin time, fibrinogen degradation product, D-dimer, creatinine, lactate, heart rate, Sequential Organ Failure Assessment, and Acute Physiology 
TABLE 1: Patient characteristics upon admission to the intensive care unit.

\begin{tabular}{|c|c|c|c|c|c|c|}
\hline \multirow{2}{*}{ Characteristic } & \multicolumn{3}{|c|}{ Training cohort } & \multicolumn{3}{|c|}{ Validation cohort } \\
\hline & Survivors $(n=112)$ & Died at 90 days $(n=49)$ & $P$ value & Survivors $(n=46)$ & Died at 90 days $(n=24)$ & $P$ value \\
\hline Men & $69(61.6)$ & $30(61.2)$ & 0.963 & $29(63.0)$ & $15(62.5)$ & 0.964 \\
\hline Age $\geq 57 \mathrm{yr}$ & $77(68.8)$ & $41(83.7)$ & 0.055 & $30(65.2)$ & $22(91.7)$ & 0.016 \\
\hline \multicolumn{7}{|l|}{ Comorbidity } \\
\hline Diabetes & $17(15.2)$ & $10(20.4)$ & 0.414 & $17(37.0)$ & $9(37.5)$ & 0.964 \\
\hline Hypertension & $42(37.5)$ & $26(53.1)$ & 0.066 & $21(45.7)$ & $12(50.0)$ & 0.729 \\
\hline COPD & $6(5.4)$ & $6(12.2)$ & 0.126 & $8(17.4)$ & $6(25.0)$ & 0.450 \\
\hline CKD & $10(8.9)$ & $6(12.2)$ & 0.518 & $2(4.3)$ & $3(12.5)$ & 0.209 \\
\hline \multicolumn{7}{|l|}{ Source of infection } \\
\hline Pulmonary & $71(63.4)$ & $33(67.3)$ & 0.629 & $27(58.7)$ & $19(71.2)$ & 0.087 \\
\hline Urinary tract & $8(7.1)$ & $1(2.0)$ & 0.195 & $5(10.9)$ & $0(0)$ & 0.094 \\
\hline Abdominal & $27(24.1)$ & $14(28.6)$ & 0.550 & $13(28.3)$ & $3(12.5)$ & 0.136 \\
\hline Skin & $6(5.4)$ & $2(4.1)$ & 0.732 & $2(4.3)$ & $1(4.2)$ & 0.972 \\
\hline $\mathrm{TM} \geq 13.1 \mathrm{TU} / \mathrm{mL}$ & $48(42.9)$ & $32(65.3)$ & 0.010 & $17(37.0)$ & $17(70.8)$ & 0.007 \\
\hline TAT (ng/mL) & $8.2(4.6-18.0)$ & $17.2(5.7-46.8)$ & 0.002 & $8.7(5.6-17.0)$ & $13.4(6.2-30.9)$ & 0.162 \\
\hline PIC $(\mu \mathrm{g} / \mathrm{mL})$ & $1.16(0.62-2.16)$ & $1.04(0.57-2.28)$ & 0.742 & $1.10(0.75-1.48)$ & $1.43(0.69-2.83)$ & 0.421 \\
\hline t-PAIC (ng/mL) & $12.2(7.6-24.1)$ & $21.7(11.3-41.7)$ & 0.003 & $14.2(9.4-23.9)$ & $21.3(13.8-47.1)$ & 0.020 \\
\hline PT (s) & $14.2(12.7-16.2)$ & $16.4(14.0-21.4)$ & 0.000 & $13.7(13-15.3)$ & 15 (13.6-19.6) & 0.008 \\
\hline INR & $1.2(1.1-1.3)$ & $1.4(1.2-1.8)$ & 0.000 & $1.14(1.08-1.27)$ & $1.25(1.13-1.60)$ & 0.008 \\
\hline $\operatorname{APTT}(\mathrm{s})$ & $31.6(26.6-38.4)$ & $37.4(32.0-47.7)$ & 0.000 & $31.4(26.7-40.5)$ & $33.9(29.2-48.7)$ & 0.087 \\
\hline FIB (g/L) & $2.9 \pm 1.09$ & $2.6 \pm 1.2$ & 0.143 & $2.9 \pm 0.9$ & $2.7 \pm 1.3$ & 0.308 \\
\hline TT (s) & $15.8(14.5-17.3)$ & $17.2(14.8-18.7)$ & 0.003 & $15.5(14.0-17.4)$ & $16.8(14.9-19.4)$ & 0.070 \\
\hline FDP $(\mu \mathrm{g} / \mathrm{L})$ & $8.69(3.67-18.92)$ & $14.45(4.53-38.00)$ & 0.030 & $7.56(4.51-13.12)$ & $11.37(6.99-27.95)$ & 0.033 \\
\hline $\mathrm{D}$-dimer $(\mu \mathrm{g} / \mathrm{L})$ & $2.59(1.03-5.97)$ & $4.91(1.65-11.00)$ & 0.016 & $2.19(0.87-4.53)$ & $3.19(2.54-7.76)$ & 0.015 \\
\hline Platelets $\left(\times 10^{9} / \mathrm{L}\right)$ & $179 \pm 90$ & $138 \pm 94$ & 0.010 & $182 \pm 108$ & $209 \pm 128$ & 0.358 \\
\hline Hemoglobin $(\mathrm{g} / \mathrm{L})$ & $111 \pm 29$ & $100 \pm 31$ & 0.038 & $109 \pm 31$ & $104 \pm 31$ & 0.525 \\
\hline ALT (U/L) & $31.9(12.9-73.5)$ & $21.5(13.3-116.8)$ & 0.597 & $27.3(13.3-58.7)$ & $29.6(11.1-64.2)$ & 0.921 \\
\hline AST (U/L) & $43.0(23.3-84.3)$ & $42.1(26.4-131.2)$ & 0.483 & $33.2(19.8-72.5)$ & $30.3(19.1-76.7)$ & 0.843 \\
\hline TBil $(\mu \mathrm{mol} / \mathrm{L})$ & $13.5(7.9-22.5)$ & $13.9(7.4-32.5)$ & 0.514 & $14.5(6.8-23.4)$ & $17.6(10.9-28.1)$ & 0.192 \\
\hline $\mathrm{Cr}(\mu \mathrm{mol} / \mathrm{mL})$ & $92.6(62.3-163.8)$ & $136(76.5-241.4)$ & 0.017 & $70.3(54.5-132.5)$ & $113.4(78.3-150.0)$ & 0.056 \\
\hline $\mathrm{RBG}(\mathrm{mmol} / \mathrm{L})$ & $7.3(6.2-9.3)$ & $6.8(5.5-9.1)$ & 0.142 & $7.6(6.7-9.6)$ & $8.8(7.1-10.5)$ & 0.239 \\
\hline Body temp $\left({ }^{\circ} \mathrm{C}\right)$ & $36.7(36.5-37.5)$ & $36.6(36.3-37.3)$ & 0.350 & $36.7(36.2-37.3)$ & $36.4(36.0-36.8)$ & 0.176 \\
\hline Heart rate $\left(\mathrm{min}^{-1}\right)$ & $96 \pm 20$ & $106 \pm 25$ & 0.013 & $98 \pm 26$ & $107 \pm 26$ & 0.179 \\
\hline MAP (mmHg) & $90 \pm 17$ & $88 \pm 22$ & 0.547 & $91 \pm 17$ & $87 \pm 18$ & 0.352 \\
\hline SOFA score & $7(5-10)$ & $9(7-15)$ & 0.000 & $7(5-10)$ & $9(6-13)$ & 0.065 \\
\hline APACHE II score & $21 \pm 6$ & $24 \pm 6$ & 0.008 & $22 \pm 7$ & $27 \pm 7$ & 0.004 \\
\hline $\mathrm{PH}$ & $7.41(7.35-7.45)$ & $7.38(7.29-7.50)$ & 0.133 & $7.42(7.34-7.49)$ & $7.29(7.19-7.43)$ & 0.006 \\
\hline $\mathrm{PaCO}_{2}(\mathrm{mmHg})$ & $36(31-42)$ & $34(29-40)$ & 0.149 & $34(28-41)$ & $39(32-46)$ & 0.040 \\
\hline $\mathrm{PaO}_{2}(\mathrm{mmHg})$ & $110(81-157)$ & $93(64-140)$ & 0.017 & $112(80-167)$ & $97.1(64-152)$ & 0.366 \\
\hline $\mathrm{Lac}(\mathrm{mmol} / \mathrm{L})$ & $1.7(1-3.2)$ & $3.2(1.5-6.6)$ & 0.000 & $2.0(1.2-3.3)$ & $3.8(1.8-9.5)$ & 0.010 \\
\hline
\end{tabular}

Values are $n(\%)$, mean \pm SD, or median (interquartile range). Abbreviations: COPD: chronic obstructive pulmonary disease; CKD: chronic kidney disease; TM: thrombomodulin; TAT: thrombin-antithrombin complex; PIC: $\alpha 2$-plasmininhibitor-plasmin complex; tPAIC: tissue plasminogen activator-inhibitor complex; PLT: platelet; HB: hemoglobin; PT: prothrombin time; APTT: activated partial thrombin time; FIB: fibrinogen; INR: international normalized ratio; TT: thrombin time; FDP: fibrinogen degradation product; ALT: alanine transaminase; AST: aspartate transaminase; TBil: total bilirubin; MAP: mean arterial pressure; SOFA: Sequential Organ Failure Assessment; APACHE II: Acute Physiology and Chronic Health Evaluation II; pH: potential of hydrogen; $\mathrm{PaO}_{2}$ : arterial partial oxygen pressure; $\mathrm{PaCO}_{2}$ : arterial partial pressure of carbon dioxide; Lac: lactate; RBG: random blood glucose.

and Chronic Health Evaluation II. Conversely, survivors showed significantly higher levels of platelet, hemoglobin, and arterial partial oxygen pressure.
3.2. Nomogram Construction. Multiple logistic regression identified age, international normalized ratio, lactate, and thrombomodulin as independent predictors of 90-day 
TABLE 2: Multivariate logistic regression of data from the training cohort to identify factors independently associated with 90 -day mortality.

\begin{tabular}{lccc}
\hline Variable & Odds ratio & 95\% confidence interval & $P$ value \\
\hline Age $(\geq 57$ vs. $<57$ y) & 1.20 & $0.36-2.04$ & 0.005 \\
TM $(\geq 13.1$ vs. $<13.1 \mathrm{TU} / \mathrm{mL})$ & 1.30 & $0.39-2.21$ & 0.005 \\
INR & 1.52 & $0.23-2.80$ & 0.021 \\
Lac $(\mathrm{mmol} / \mathrm{L})$ & 0.17 & $0.04-0.29$ & 0.008 \\
\hline
\end{tabular}

INR: international normalized ratio; TM: thrombomodulin; Lac: lactate.

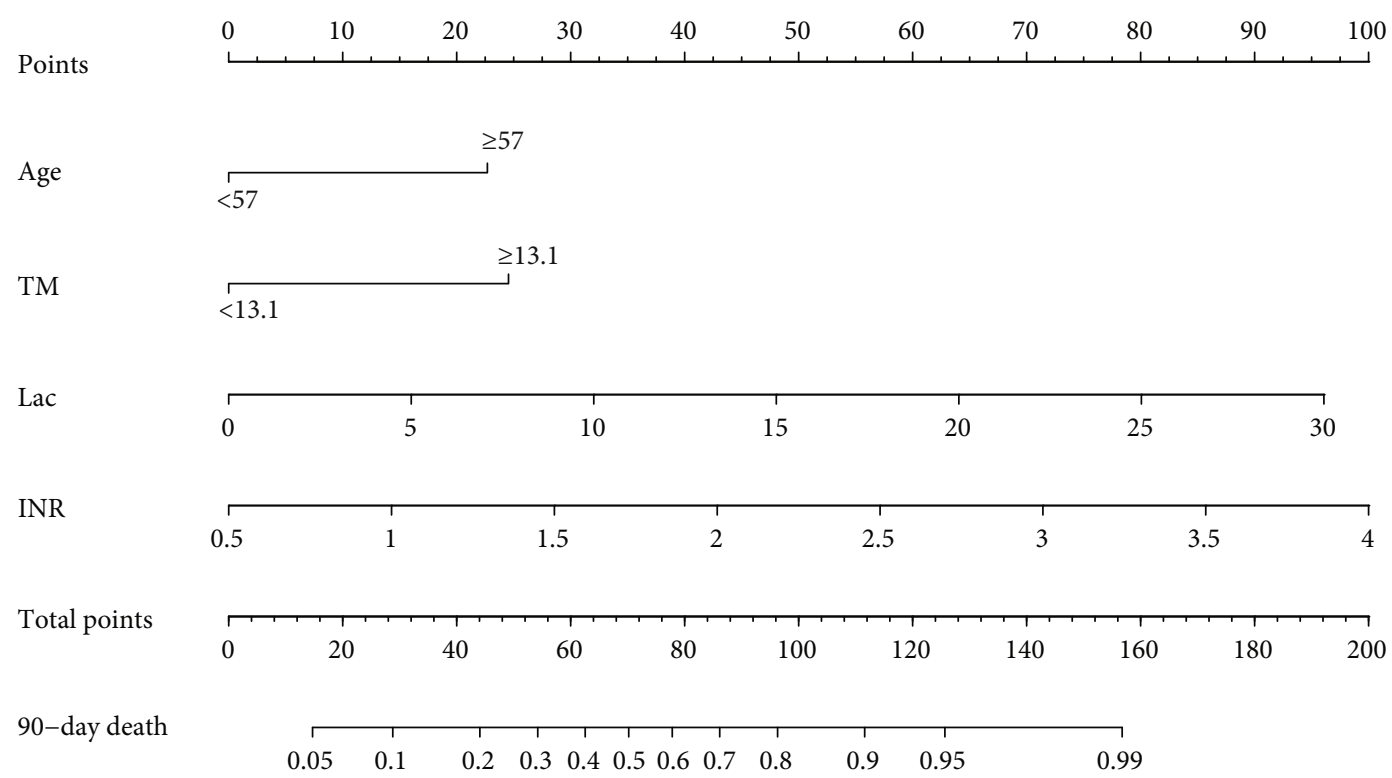

FIgURE 2: Nomogram for predicting 90-day mortality in patients with sepsis, based on data in the training cohort.

mortality (Table 2), which were then integrated into a predictive nomogram (Figure 2). The results of regression analysis were visualized. The clinician can give an individualized evaluation of the risk of 90-day mortality for patients undergoing sepsis according to the total points which were obtained by adding each score in the nomogram. This would facilitate precise risk assessment and better identification of 90-day mortality in the septic population.

3.3. Nomogram Validation. The nomogram based on data in the training cohort gave an AUC of 0.810 (95\% CI 0.739 to 0.881 ) for predicting 90-day mortality in that cohort (Figure 3(a)). Similarly, it gave an AUC of 0.813 (95\% CI 0.708 to 0.917 ) for predicting 90 -day mortality in the validation cohort (Figure 3(b)).

For both cohorts, the nomogram showed good agreement with actual 90-day mortality based on calibration curves (Figure 4), although the logistic calibration curve and nonparametric curve deviated slightly from the ideal line. The Hosmer-Lemeshow test gave a $P=0.866$ in the training cohort while it gave a $P=0.801$ in the validation cohort, suggesting no significant deviation from a perfect fit.

3.4. Potential Clinical Usefulness of the Nomogram. DCA showed good clinical potential for the nomogram, based on the training cohort (Figure 5(a)) and validation cohort
(Figure 5(b)). When the threshold probability is greater than $15 \%$, using the nomogram can lead to lower mortality than treating either all or none of the patients.

\section{Discussion}

In this study, we defined a nomogram based on routinely measured clinical variables that may reliably predict 90-day mortality among patients with sepsis. While our nomogram should be verified with other patient populations, it establishes the feasibility of accurate mortality prediction using relatively simple clinical tests. While several studies have identified risk factors associated with 90-day mortality in sepsis, our work suggests that certain risk factors may be particularly relevant for screening patients for mortality risk.

The 90-day mortality in our retrospective cohort of Chinese patients was $31.6 \%$, which was higher than that in previous studies $[2,3,5]$. Sepsis patients concluded in the present study had much higher APACHE II scores and had a longer follow-up (90-day mortality) than those in previous reports, which could explain these differences [14].

We found that the international normalized ratio was significantly higher among sepsis patients who died within 90 days of follow-up than among those who did not die, and it emerged as an independent predictor of 90-day mortality in multivariate analysis. Coagulopathy is frequently 


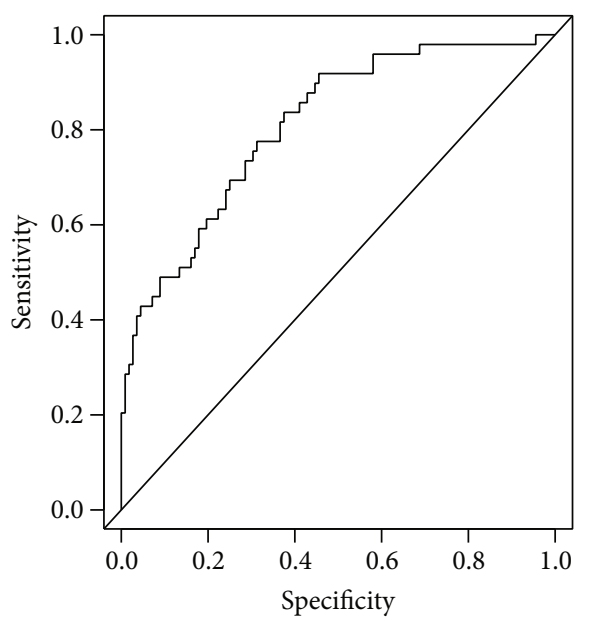

(a)

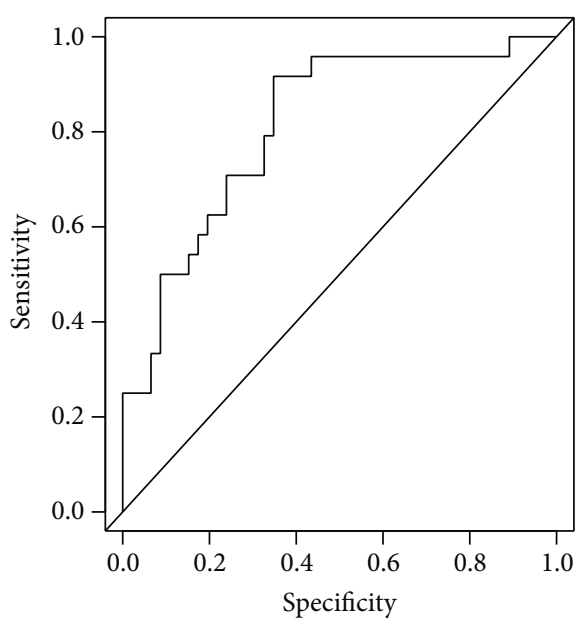

(b)

FIGURE 3: Receiver operating characteristic curves assessing the ability of the nomogram to predict 90-day mortality in (a) training and (b) validation cohorts.

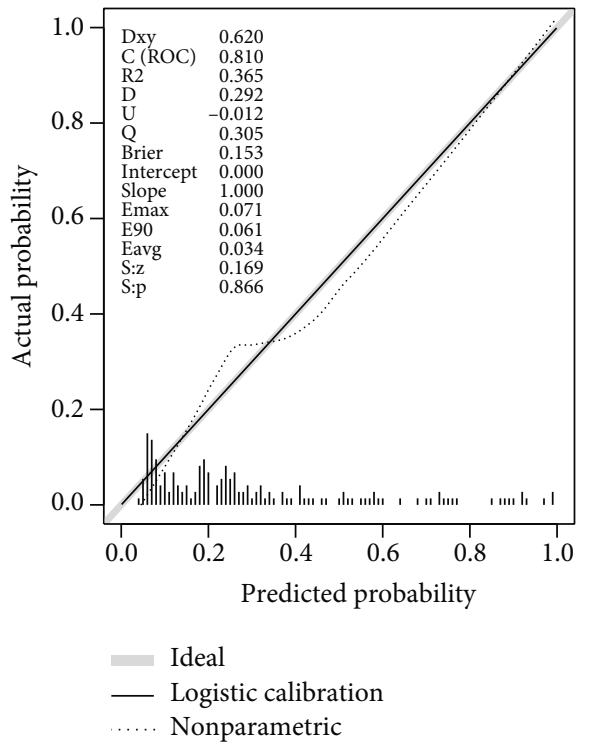

(a)

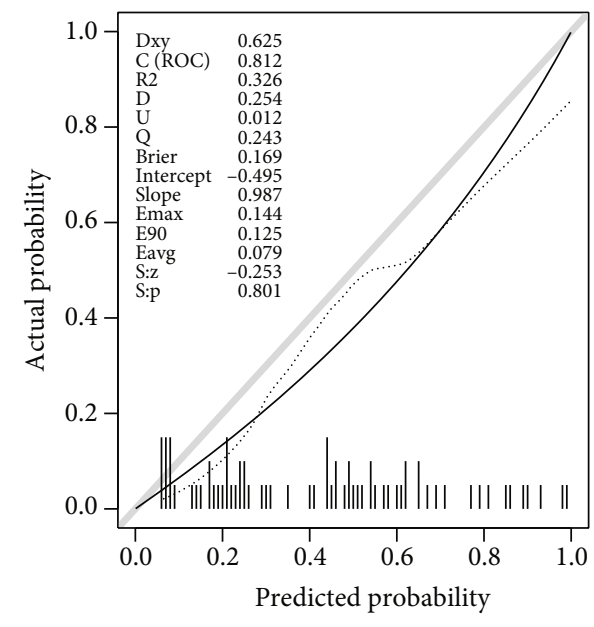

(b)

FIGURE 4: Calibration plot of predicted and observed probabilities of 90-day mortality in (a) training and (b) validation cohorts.

observed in sepsis [15], and it contributes to multiple organ dysfunction syndrome [16]. More severe coagulopathy has been linked to higher risk of mortality among patients with sepsis [17], and clinical parameters reflecting hemostasis can predict sepsis-related mortality [18-20]. Our results are consistent with this literature. Nevertheless, the international normalized ratio alone cannot accurately predict sepsis outcomes [5], which may be due to the need to take into account other independent predictors of mortality.

One of those predictors is lactate level, which was significantly higher among our patients who died within 90 days than among those who did not. Critically ill patients, particularly those with sepsis or septic shock, show elevated lactate [21], and the magnitude of the elevation correlates strongly and positively with sepsis severity and associated mortality
[22-24]. Serum lactate levels are considered a marker of tissue hypoxia [19], and they have proven useful for guiding clinical treatment and predicting prognosis in various clinical contexts [25]. Our study supports the "Sepsis-3" recommendation that septic shock should be defined as persistence of serum lactate $>2 \mathrm{mmol} / \mathrm{L}[10]$.

Another risk factor for 90-day mortality that emerged as particularly important for prediction was elevated thrombomodulin level. Thrombomodulin, an integral endothelial cell membrane protein, is cleaved and released into the bloodstream during sepsis and septic shock [26, 27], leading to elevated levels of serum thrombomodulin in pediatric and adult sepsis patients $[28,29]$. Endothelium is the primary site of damage in sepsis due to massive production of proinflammatory cytokines [6]. Elevated serum thrombomodulin 


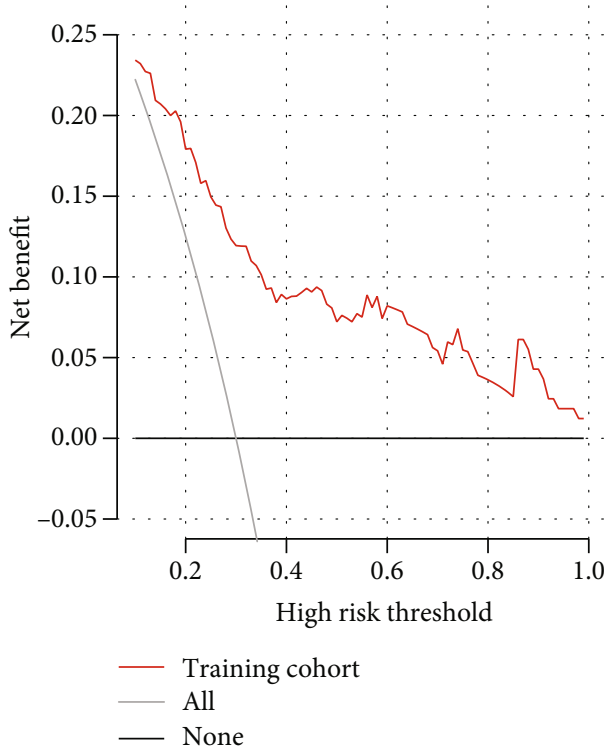

(a)

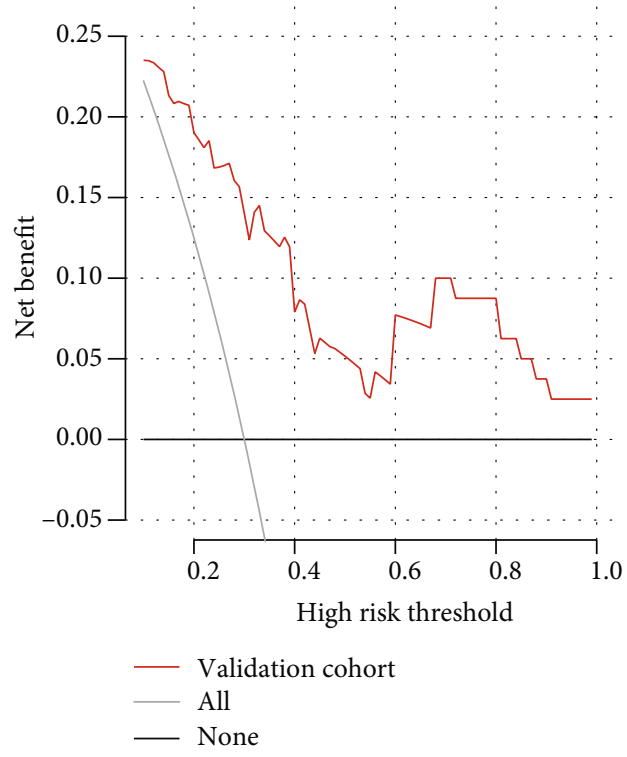

(b)

FIGURE 5: Decision curve analysis to assess the benefit of clinical intervention based on the predictive nomogram in (a) training and (b) validation cohorts.

level is associated with sepsis severity and risk of death [30]. Our study showed that endothelial cell injury justified by elevated TM activated the coagulation system, depleted coagulation factors characterized by prolonged PT to promote microthrombosis, and caused tissue hypoperfusion and increased lactate, especially obviously in elder patients with sepsis.

Our nomogram showed AUC values above 0.8 for the training and validation cohorts, suggesting good predictive ability. In addition, DCA suggested that treating our cohorts according to our nomogram's predictions could be superior to treating all or none of them. The calibration curve also suggested good fit. Nevertheless, our model was generated based on retrospective analysis of a relatively small sample from a single medical center, so it should be validated in other patient populations. It may be possible to further improve the model by a multicenter study with external validation.

\section{Conclusions}

We have developed a nomogram that may reliably predict 90-day mortality in patients with sepsis, based on age, international normalized ratio, lactate, and thrombomodulin. This may help clinicians identify patients at higher risk and modify clinical management and resource allocation accordingly.

\section{Data Availability}

The raw data supporting the conclusions of this article will be made available by the authors, without undue reservation.

\section{Ethical Approval}

The study was approved by the Ethics Committee of all the study centers.

\section{Consent}

Written informed consent was waived by the Ethics Commission of 908th hospital because of the retrospective nature of this study.

\section{Conflicts of Interest}

The authors declare that they have no competing interests.

\section{Authors' Contributions}

QBZ, LPH, and JCS designed this study. LPH collected the data. NQZ, LCZ, and QWL were responsible for the statistical analysis. QBZ and LPH were the first writing authors of the manuscript. JCS finalized this manuscript. The authors read and approved the final manuscript. Qing-Bo Zeng and Long-Ping He contributed equally to this work, and they are the co-first authors.

\section{Acknowledgments}

The manuscript has been released as a preprint for communication and sharing with peers.

\section{References}

[1] A. Rhodes, L. E. Evans, W. Alhazzani et al., "Surviving sepsis campaign: international guidelines for management of sepsis and septic shock: 2016," Critical Care Medicine, vol. 43, no. 3, pp. 304-377, 2017. 
[2] C. Fleischmann, A. Scherag, N. K. Adhikari et al., “Assessment of global incidence and mortality of hospital-treated sepsis. Current Estimates and Limitations," American Journal of Respiratory and Critical Care Medicine, vol. 193, no. 3, pp. 259-272, 2016.

[3] J. Xie, H. Wang, Y. Kang et al., "The epidemiology of sepsis in Chinese ICUs: a national cross-sectional survey," Critical Care Medicine, vol. 48, no. 3, pp. e209-e218, 2020.

[4] M. Huang, S. Cai, and J. Su, "The pathogenesis of sepsis and potential therapeutic targets," International Journal of Molecular Sciences, vol. 20, no. 21, p. 5376, 2019.

[5] J. Liu, C. Bai, B. Li et al., "Mortality prediction using a novel combination of biomarkers in the first day of sepsis in intensive care units," Scientific Reports, vol. 11, no. 1, p. $1275,2021$.

[6] M. E. Johansen, P. I. Johansson, S. R. Ostrowski et al., "Profound endothelial damage predicts impending organ failure and death in sepsis," Seminars in Thrombosis and Hemostasis, vol. 41, no. 1, pp. 16-25, 2015.

[7] M. Wang, L. Jiang, B. Zhu et al., "The prevalence, risk factors, and outcomes of sepsis in critically ill patients in China: a multicenter prospective cohort study," Frontiers of Medicine (Lausanne), vol. 7, article 593808, 2020.

[8] V. D’Onofrio, A. Meersman, S. Vijgen, R. Cartuyvels, P. Messiaen, and I. C. Gyssens, "Risk factors for mortality, ICU admission and bacteremia in patients suspected of sepsis at the emergency department. a prospective cohort study," Open Forum Infectious Diseases, vol. 8, no. 1, p. ofaa594, 2020.

[9] F. Innocenti, F. Meo, I. Giacomelli et al., "Prognostic value of serial lactate levels in septic patients with and without shock," Internal and Emergency Medicine, vol. 14, no. 8, pp. 13211330, 2019.

[10] M. Singer, C. S. Deutschman, C. W. Seymour et al., "The Third International Consensus Definitions for Sepsis and Septic Shock (Sepsis-3)," Journal of the American Medical Association, vol. 315, no. 8, pp. 801-810, 2016.

[11] W. A. Knaus, E. A. Draper, D. P. Wagner, and J. E. Zimmerman, "APACHE II: a severity of disease classification system," Critical Care Medicine, vol. 13, no. 10, pp. 818-829, 1985.

[12] E. P. Raith, A. A. Udy, M. Bailey et al., "Prognostic accuracy of the SOFA score, SIRS criteria, and qSOFA score for in-hospital mortality among adults with suspected infection admitted to the intensive care unit," Journal of the American Medical Association, vol. 317, no. 3, pp. 290-300, 2017.

[13] G. S. Collins, J. B. Reitsma, D. G. Altman, and K. G. Moons, "Transparent reporting of a multivariable prediction model for individual prognosis or diagnosis (TRIPOD): the TRIPOD statement," British Medical Journal, vol. 350, no. jan07 4, p. g7594, 2015.

[14] K. M. Kaukonen, M. Bailey, S. Suzuki, D. Pilcher, and R. Bellomo, "Mortality related to severe sepsis and septic shock among critically ill patients in Australia and New Zealand, 2000-2012," Journal of the American Medical Association, vol. 311, no. 13, pp. 1308-1316, 2014.

[15] A. Perner, A. Rhodes, B. Venkatesh et al., "Sepsis: frontiers in supportive care, organisation and research," Intensive Care Medicine, vol. 43, no. 4, pp. 496-508, 2017.

[16] C. Lelubre and J. L. Vincent, "Mechanisms and treatment of organ failure in sepsis," Nature Reviews Nephrology, vol. 14, no. 7, pp. 417-427, 2018.
[17] P. G. Lyons, S. T. Micek, N. Hampton, and M. H. Kollef, "Sepsis-associated coagulopathy severity predicts hospital mortality," Critical Care Medicine, vol. 46, no. 5, pp. 736-742, 2018.

[18] F. Innocenti, A. M. Gori, B. Giusti et al., "Prognostic value of sepsis-induced coagulation abnormalities: an early assessment in the emergency department," Internal and Emergency Medicine, vol. 14, no. 3, pp. 459-466, 2019.

[19] S. Benediktsson, A. Frigyesi, and T. Kander, "Routine coagulation tests on ICU admission are associated with mortality in sepsis: an observational study," Acta Anaesthesiologica Scandinavica, vol. 61, no. 7, pp. 790-796, 2017.

[20] T. Iba, M. D. Nisio, J. H. Levy, N. Kitamura, and J. Thachil, "New criteria for sepsis-induced coagulopathy (SIC) following the revised sepsis definition: a retrospective analysis of a nationwide survey," British Medical Journal Open, vol. 7, no. 9, article e017046, 2017.

[21] B. Suetrong and K. R. Walley, "Lactic acidosis in sepsis: it's not all anaerobic: implications for diagnosis and management," Chest, vol. 149, no. 1, pp. 252-261, 2016.

[22] B. Nolt, F. Tu, X. Wang et al., "Lactate and immunosuppression in sepsis," Shock, vol. 49, no. 2, pp. 120-125, 2018.

[23] H. B. Nguyen, E. P. Rivers, B. P. Knoblich et al., "Early lactate clearance is associated with improved outcome in severe sepsis and septic shock," Critical Care Medicine, vol. 32, no. 8, pp. 1637-1642, 2004.

[24] P. Wacharasint, T. A. Nakada, J. H. Boyd, J. A. Russell, and K. R. Walley, "Normal-range blood lactate concentration in septic shock is prognostic and predictive," Shock, vol. 38, no. 1, pp. 4-10, 2012.

[25] A. H. Rishu, R. Khan, H. M. al-Dorzi et al., "Even mild hyperlactatemia is associated with increased mortality in critically ill patients," Critical Care, vol. 17, no. 5, p. R197, 2013.

[26] M. Schouten, W. J. Wiersinga, M. Levi, and T. van der Poll, "Inflammation, endothelium, and coagulation in sepsis," Journal of Leukocyte Biology, vol. 83, no. 3, pp. 536-545, 2008.

[27] C. T. Esmon and W. G. Owen, "Identification of an endothelial cell cofactor for thrombin-catalyzed activation of protein C," proceedings of the national academy of sciences of the United States of America, vol. 78, no. 4, pp. 2249-2252, 1981.

[28] Z. Liu, Z. Meng, Y. Li et al., "Prognostic accuracy of the serum lactate level, the SOFA score and the qSOFA score for mortality among adults with sepsis," Scandinavian journal of trauma, resuscitation and emergency, vol. 27, no. 1, p. 51, 2019.

[29] J. J. Lin, H. J. Hsiao, O. W. Chan, Y. Wang, S. H. Hsia, and C. H. Chiu, "Increased serum thrombomodulin level is associated with disease severity and mortality in pediatric sepsis," PLoS One, vol. 12, no. 8, article e0182324, 2017.

[30] S. M. Lin, Y. M. Wang, H. C. Lin et al., "Serum thrombomodulin level relates to the clinical course of disseminated intravascular coagulation, multiorgan dysfunction syndrome, and mortality in patients with sepsis," Critical Care Medicine, vol. 36, no. 3, pp. 683-689, 2008. 